\title{
Un Modelo de Programación No-lineal para la Planeación de la Producción de Gas y Petróleo
}

\author{
Oswaldo Robles-Agudo y Richart Vázquez-Román* \\ Instituto Tecnológico de Celaya, Departamento de Ingeniería Química, Avenida Tecnológico y \\ García Cubas s/n, 38010 Celaya, Gto.-México (e-mail: richart@iqcelaya.itc.mx)
}

*autor a quien debe ser dirigida la correspondencia

\section{Resumen}

En este trabajo se propone un modelo matemático que permite optimizar la planeación de la producción de gas y petróleo de varios pozos. El horizonte de planeación se divide en varios períodos de tiempo en los cuales se tiene una demanda conocida y se asocia un coeficiente de costo a cada pozo en la función objetivo. La formulación contempla la disminución de la presión en la boca del pozo debido a la extracción y la recuperación de la presión cuando la producción del mismo se anula. Además, se modela las restricciones debidas a la caída de presión en las tuberías y la ínterconectividad de los pozos. Los resultados indican que el modelo es capaz de planear la producción sin necesidad de formular el caso como un problema de programación no-lineal mixta entera. no-lineal

\section{A Non-Linear Programming Model for the Gas and Oil Production Planning}

\begin{abstract}
In this work, a model to optimize the planning of gas and oil production in several wells is proposed. The planning horizon is divided in several periods for which the demand is known, and a cost coefficient is associated to each well in the objective function. The formulation includes the pressure decrease effect at the wellbore when the well is producing and the pressure increase when the well is not producing. In addition, constrains to the flow and the wells interconnectivity are also incorporated in the model. The results show that the proposed model can be used in planning oil and gas production without using mixed integer non-linear programming.
\end{abstract}

Keywords: gas production, oil production, planning systems, non-linear programming 


\section{INTRODUCCIÓN}

Un sistema de producción típico de gas y/o petróleo consiste en al menos un yacimiento que contiene una o varias secciones en donde varios pozos han sido perforados para la producción o incluso inyección de hidrocarburos. El punto de entrada de gas o petróleo al pozo se denomina como boca del pozo. Los recursos en la superficie forman también parte del sistema de producción. En general, los pozos de uno o más yacimientos se interconectan a través de cabezales para transferir la producción global a través de una sola tubería que será llamada colector en este trabajo. Las válvulas representan una parte importante en los sistemas de producción ya que permiten racionar el flujo individual de cada pozo. Así, la red de la tubería resultante incluye yacimientos, pozos, colectores, y válvulas (Fig. 1). Los yacimientos pueden visualizarse como contenedores dinámicos ya que la composición y condiciones de presión y temperatura cambian tan pronto se inicia la producción (Zhu et al., 2003). Afortunadamente, los cambios en composición y temperatura son perceptibles sólo en tiempos razonablemente grandes. La presión es una variable que limita frecuentemente la producción. La explotación excesiva en un pozo hace que la presión en la boca del pozo disminuya al no recuperarse con el hidrocarburo del yacimiento debido a la lentitud con que fluye a través del medio poroso circundante. Esta disminución conlleva a la falta del hidrocarburo o a la falta de energía para extraer el hidrocarburo existente. El uso de algún tipo de levantamiento mecánico (bombas) o neumático es característico del sector petrolero cuando la producción no se logra de forma natural (Economides et al., 1994). Otras operaciones adicionales tendientes a mantener la producción de petróleo incluyen las recuperaciones primarias y las varias recuperaciones mejoradas del petróleo (Green y Willhite, 1998).

La literatura reporta varios trabajos relacionados con la simulación, optimización y planeación de sistemas de producción para demostrar aspectos específicos, incluyendo simplificaciones, del sistema. Por ejemplo, el cálculo del flujo desde el yacimiento hasta la boca del pozo se ha simplificado a través de modelos lineales (Horne, 1998). La simulación de un sistema de producción se ha basado en gran medida en el denominado análisis NODAL (Beggs, 2003). La simulación del proceso se ha resuelto usando un método de optimización que ha sido comercializado por Edinburgh Petroleum Services (Handley-Schachler et al., 2000). Recientemente, la simulación incluyó ecuaciones de estado cúbicas para la estimación de propiedades, generando un modelo riguroso de ecuaciones diferencialalgebraicas (Barragán-Hernández et al., 2005). El problema de optimización ha recibido particular atención por varios investigadores quienes han orientado sus trabajos a diversos aspectos. Después de lograr la simulación del proceso, una de las extensiones al modelo consiste en definir la estrategia de producción diaria (Heinemann et al., 1998). En este caso se asume que las variables que caracterizan al yacimiento permanecen lo suficientemente invariantes. Las limitantes a la producción incluyen las relacionadas a la representación física del sistema como son el diámetro, profundidad y longitud. En particular, la ínterconectividad debe ser claramente definida ya que la posibilidad de inyección en el cabezal depende de los pozos interconectados. Un modelo que incluye pozos con levantamiento neumático ha sido resuelto con la implementación de conjuntos ordenados especiales (Kosmidis et al., 2004). Los mismos autores proponen un modelo de programación no-lineal mixta entera (MINLP por sus siglas en inglés) cuyo balance de energía y estimación de propiedades han sido simplificados (Kosmidis et al., 2005). Las variables discretas son propuestas en ese trabajo para incorporar la ínterconectividad de los pozos. En general, una gran cantidad de trabajos publicados resuelven la simulación de la producción del petróleo y su optimización a través de reglas heurísticas.

La planeación óptima de la producción es un área relativamente nueva. La programación lineal se ha usado para resolver la planeación de algunos esquemas coordinados en la producción de petróleo (Eeg y Herring, 1997). La estrategia MINLP fue aplicada en la planificación de múltiples periodos incorporando algunos aspectos legales como los impuestos (lyer et al., 1998). Otro método MINLP fue aplicado para planear la producción del yacimiento petrolífero a nivel gerencial (Van den Heever y Grossmann, 2000). En este caso, se usa una técnica de descomposición de bilevel con agregación de períodos de tiempo y permitiendo la inclusión de impuestos y regalías en la función objetivo. Un análisis extenso del problema y un acercamiento innovador basado en una descomposición heurística del Lagrangiano se presentó en (Van den Heever et al., 2001). Un modelo para la planeación de la producción de petróleo ha sido también desarrollado (Ortíz-Gómez et al., 2001). Una planeación táctica de la producción incluyendo interrupciones en la producción ha sido publicada recientemente 
(Ulstein et al., 2007). Un algoritmo evolucionario para optimización de objetivo múltiple ha sido aplicado en la planeación de la producción con sistemas de levantamiento neumático (Tapabrata y Sarker, 2007).

La revisión bibliográfica indica que los trabajos reportados en la literatura tienden a combinar variables continuas con discretas para resolver el problema de planeación. La principal desventaja de estos métodos es la lentitud en la solución del problema cuando el número de pozos aumenta.

En este trabajo se desarrolla un modelo de programación no-lineal (NLP) para resolver el problema de la planeación sin recurrir al modelado MINLP. La estrategia de modelado aplicada permite la aproximación de la discretización evitando el crecimiento exponencial del tiempo en la solución numérica.

\section{MODELO MATEMÁTICO}

La descripción del sistema adoptado en este trabajo es idéntico al usado en Barragán-Hernández et al. (2005). Así, el sistema de producción consiste de un conjunto de pozos interconectados a través de colectores. En el problema a resolver, se asume que la demanda no es constante y se distribuye en diversos periodos de tiempo. Sin embargo, la demanda se asume constante en cada período de tiempo.

La producción de un pozo se mantiene constante durante cada período y su capacidad de producción es restringida por varios factores. La primera restricción involucra el comportamiento de la boca del pozo con respecto al yacimiento. Varios modelos han sido propuestos para modelar diferentes tipos de pozos lo cual incluye la influencia de pozos vecinos, fracturas, etc. (Economides et al., 1994). Para demostrar la factibilidad de este trabajo se eligió sólo uno de ellos. Así, el decremento de la presión en la boca del pozo debido a la extracción, $D_{w t}$, se calcula con:

$D_{w t}=q_{w t}\left\{c_{1 w}\left[\ln T+c_{2 w}\right]\right\}$

donde $q_{w t}$ es el flujo extraído, $T$ es el tiempo total del período y $c_{1 w}$ y $c_{2 w}$ son constantes determinadas experimentalmente. En el sector petrolero se usan unidades particulares que pueden ser transformadas fácilmente a cualquier sistema modificando las constantes $c_{1 w}$ y $c_{2 w}$.

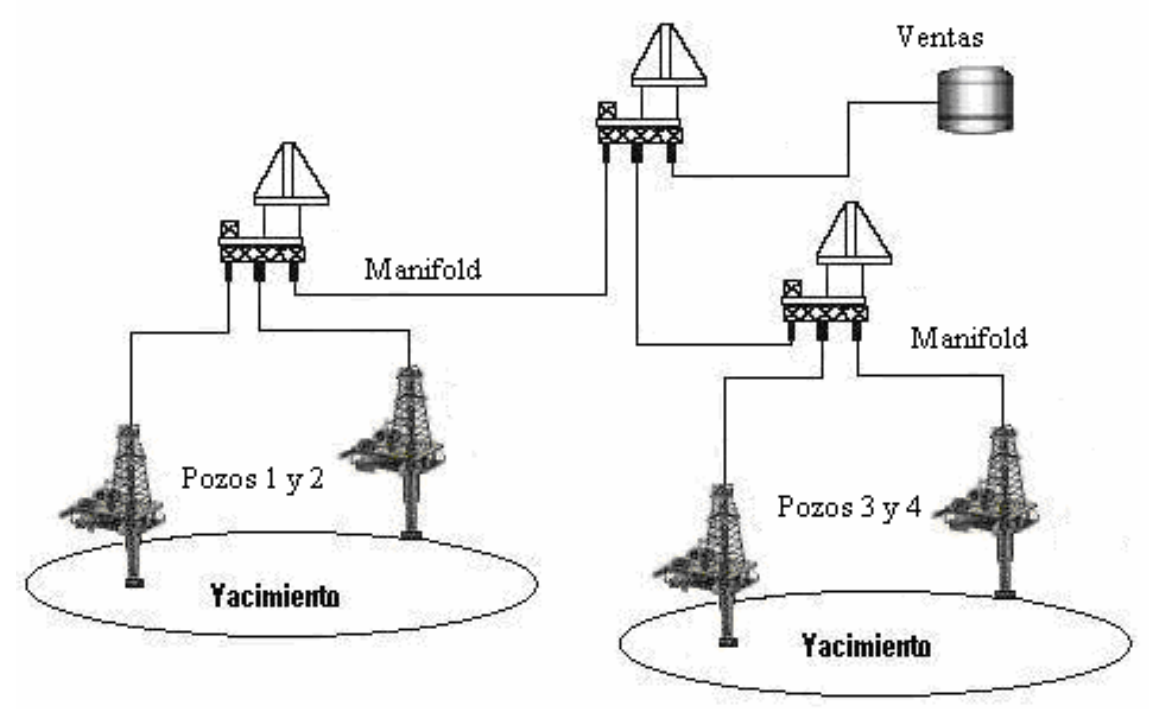

Fig. 1: Sistemas de producción de gas y petróleo 
Cuando el pozo no produce, la presión en la boca del pozo se incrementa de acuerdo a:

$I_{w t}=\left\{c_{1 w}^{r e c}\left[\ln (T)+c_{2 q}^{r e c}\right]\right\}$

donde $I_{w t}$ es el incremento y $c_{1 w}^{r e c}$ y $c_{2 q}^{r e c}$ son constantes para cada pozo.

Adicionalmente, el flujo máximo a extraerse de un pozo debido a las propiedades geológicas, $q_{w t}^{\max }$, puede calcularse como:

$q_{w t}^{\max }\left\{c_{1 t}\left[\ln (T)+c_{2}\right]\right\}=\left(P_{w t}^{i n}-P_{w}^{l o w}\right)$

donde $P_{w t}^{\text {in }}$ es la presión en la boca del pozo $w$ al inicio del período $t, c_{1 t}, c_{2}$ y $P_{w}^{\text {low }}$ son constantes determinadas experimentalmente. Así, la producción de un pozo se limita a un flujo menor o igual al flujo máximo permitido por las propiedades geológicas.

Una segunda restricción del flujo esta definida por la tubería que conforma el pozo así como los accesorios como válvulas y codos. Varios modelos han sido desarrollados para relacionar el flujo en la tubería con su correspondiente caída de presión. En este trabajo se usa la simplificación obtenida cuando se asume velocidad constante (Vázquez-Román, 1998). El efecto de los accesorios son incorporados en una sola constante, $C v_{w}$, lo que reduce el balance de presiones a:

$C v_{w} q_{w t}+A p_{w t} f_{w t} * c_{3 w} * q_{w t}{ }^{2}+c_{4 w}-P_{w t}^{\text {in }}+P_{w}^{\text {out }}=0$

donde $c_{3 w}, y c_{4 w}$ son parámetros que representan $g \rho L \operatorname{sen} \theta / g_{C} \quad$ y $L /\left(2 A^{2} \rho D\right)$ respectivamente, $A p_{w t}$ es la apertura de la válvula del pozo w en el periodo de tiempo $t, f_{w t}$ es el factor de fricción y $P_{w t}^{o u t}$ es la presión al final de la tubería del pozo w en el periodo t.

Cada colector, $m$, es modelado de manera semejante a lo indicado para las tuberías de los pozos:

$$
C v_{m} q_{m t}+f_{m t} * c_{5 m} * q_{m t}^{2}+c_{6 m}-P_{m t}^{i n}+P_{m}^{\text {out }}=0
$$

donde $P_{m t}^{\text {in }}$ y $P_{m t}^{\text {out }}$ son las presiones inicial y final del colector $m$ en el periodo $t, c_{5 m}$ y $c_{6 m}$ son los parámetros que representan $g \rho L \operatorname{sen} \theta / g_{C}$ y $L / 2 A^{2} \rho D$ respectivamente, y $f_{m t}$ es el factor de fricción para el pozo $w$ en el periodo $t$ y para el colector $m$.

Para, entre otras cosas, evitar la producción excesiva de arenas, el flujo y la presión en la boca del pozo se restringen de la manera siguiente:

$q_{w t} \leq q_{w t}^{\max }$

$P_{w}^{\text {low }} \leq P_{w t}^{f} \leq P_{w}^{u p}$ 
donde $P^{u p}$ es el límite superior para indicar la presión máxima que tendrá la boca del pozo la cual es generalmente igual a la presión inicial del yacimiento cuando se empieza la explotación, y $P^{\text {low }}$ es el límite inferior definido por el usuario para indicar la presión mínima que tendrá la boca del pozo.

Por otro lado, la presión en la boca del pozo al final de un período y al inicio del siguiente periodo debe ser la misma para garantizar la continuidad en el tiempo:

$P_{w t}^{i n}=P_{w t-1}^{f}$

Adicionalmente, la presión de salida del pozo debe ser igual a la presión en el punto de entrada de la tubería del colector que lo esta interconectando con otros pozos:

$P_{w}^{\text {out }}=P_{m}^{\text {in }}$

Así, la presión al final del período de tiempo se comportaría como:

$p_{w t}^{f}-p_{w t}^{i n}+D_{w t}-I_{w t}\left(1-\frac{q_{w t}}{q_{w t}+e p}\right)=0$

donde ep es una constante que impide la división por cero cuando el flujo es nulo y el cociente $q_{w t} /\left(q_{w t}+e p\right)$ tiene como función impedir el incremento de la presión en la boca del pozo cuando hay flujo.

Un balance de masa es requerido en la entrada de los colectores para permitir el mezclado de las diferentes corrientes que interconectará.

$q_{m t}=\sum_{w} q_{w t}+\sum_{m} q_{m t}$

Finalmente, la función objetivo usada en este trabajo consiste minimizar los costos totales de producción:

Min costo $=\sum_{w} \sum_{t} \gamma_{w t} q_{w t} \cdot L T+\sum_{w} \sum_{t} \delta_{w t} \cdot y_{w t} \cdot L T+\sum_{w} \sum_{t} I_{w t}$

donde $\delta$ y $\gamma$ son coeficientes de costos.

Asumiendo que las presiones del yacimiento y en los puntos de venta son conocidas, el problema de la planeación óptima de la producción tiene como variables a determinar los flujos de los pozos en cada período y las presiones intermedias. Los resultados requeridos involucran a los perfiles de producción de petróleo en cada periodo de tiempo para satisfacer la demanda impuesta también para cada periodo de tiempo. El análisis de grados de libertad realizado en (Barragán-Hernández et al., 2005) no difiere significativamente del resultado que se obtiene en este modelo. 
Tabla 1: Resultados para diferentes configuraciones

\begin{tabular}{|c|c|c|c|c|c|}
\hline Configuración & $\begin{array}{l}\text { Demanda ba- } \\
\text { rriles/semana }\end{array}$ & $\begin{array}{c}\text { Costo total en } \\
\text { USD }\end{array}$ & Cpu (seg) & Pozo -periodo & Flujo extraído \\
\hline $4 W-3 M$ & $\begin{array}{l}4000 \\
7000\end{array}$ & 27836 & 0.3 & $\begin{array}{l}\text { W1T1 } \\
\text { W2T1 } \\
\text { W3T1 } \\
\text { W4T1 } \\
\text { W1T2 } \\
\text { W2T2 } \\
\text { W3T2 } \\
\text { W4T2 }\end{array}$ & $\begin{array}{c}3386.3 \\
12.1 \\
12.1 \\
589.5 \\
3012.8 \\
12.1 \\
12.0 \\
3963.1\end{array}$ \\
\hline $6 W-4 M$ & $\begin{array}{l}6000 \\
9000\end{array}$ & 20206 & 1.3 & $\begin{array}{l}\text { W1T1 } \\
\text { W2T1 } \\
\text { W3T1 } \\
\text { W4T1 } \\
\text { W5T1 } \\
\text { W6T1 } \\
W 1 T 2 \\
\text { W2T2 } \\
\text { W3T2 } \\
\text { W4T2 } \\
\text { W5T2 } \\
W 6 T 2\end{array}$ & $\begin{array}{c}136.6 \\
117.8 \\
117.8 \\
3102.6 \\
1260.9 \\
1264.9 \\
136.5 \\
117.8 \\
117.8 \\
2343.9 \\
3834.8 \\
2449.3\end{array}$ \\
\hline $5 W-1 M$ & $\begin{array}{l}4300 \\
6500\end{array}$ & 23363 & 0.16 & $\begin{array}{l}\text { W1T1 } \\
\text { W2T1 } \\
\text { W3T1 } \\
\text { W4T1 } \\
\text { W5T1 } \\
\text { W1T2 } \\
\text { W2T2 } \\
\text { W3T2 } \\
\text { W4T2 } \\
\text { W5T2 }\end{array}$ & $\begin{array}{c}0 \\
12.1 \\
288.3 \\
0 \\
3999.5 \\
14.0 \\
12.10 .06 \\
1371.8 \\
5102.2 \\
0\end{array}$ \\
\hline $3 W-3 M$ & $\begin{array}{l}4000 \\
2500\end{array}$ & 15909 & 0.46 & $\begin{array}{l}\text { W1T1 } \\
\text { W2T1 } \\
\text { W3T1 } \\
\text { W1T2 } \\
\text { W2T2 } \\
\text { W3T2 }\end{array}$ & $\begin{array}{c}3386.5 \\
19.1 \\
594.5 \\
2492.2 \\
7.651 \\
0.175\end{array}$ \\
\hline $2 W-3 M$ & $\begin{array}{l}3300 \\
2200\end{array}$ & 13406 & 0.1 & $\begin{array}{l}\text { W1T1 } \\
W 2 T 1 \\
W 1 T 2 \\
W 2 T 2\end{array}$ & $\begin{array}{c}3287.9 \\
12.10 .06 \\
2187.9 \\
12.1\end{array}$ \\
\hline
\end{tabular}

\section{RESULTADOS Y DISCUSIÓN}

El modelo matemático desarrollado en la sección anterior fue incorporado en el sistema GAMS (Brooke et al., 1998). Primeramente se seleccionaron los métodos CONOPT, MINOS y kNITRO y se decidió detectar el mejor de ellos en términos de cpu. Se uso el caso de 15 pozos interconectados a un solo colector para satisfacer una demanda de 27,000 barriles en el primer período, 35000 barriles para el segundo periodo y 28000 barriles para el tercer periodo. Los períodos consideran una semana de operación. Los resultados obtenidos indicaron 4.5, 9.5 y 0.6 segundos usando CONOPT, MINOS y kNITRO respectivamente. 
Posteriormente, se decidió aplicar el modelo usando kNITRO para resolver el problema NLP. Para ello se propusieron varios casos de estudio en los cuales se usaron pozos con profundidades entre 4,000 y $10,000 \mathrm{~m}$, diámetros entre $0.09-0.15 \mathrm{~m}$, presiones entre 100-200 bar, y temperaturas entre $100-200^{\circ} \mathrm{C}$. Las propiedades termodinámicas requeridas fueron calculadas usando la ecuación de Peng-Robinson. Una síntesis de los resultados obtenidos se muestra en la Tabla 1. La configuración indica el número de pozos y colectores considerados en cada ejemplo, la demanda es indicada por período y los resultados indican el costo total, tiempo requerido para realizar el cálculo y la producción por pozo en cada período.

Los resultados claramente ratifican la posibilidad de realizar una planeación de la producción de gas o petróleo sin resolver un sistema de programación no-lineal mixta entera. La ventaja inmediata es la rapidez con la cual se resuelve el problema de optimización ya que únicamente se resuelve el programa no-lineal. En términos del tiempo computacional, el requerido para resolver el problema no lineal es semejante al requerido al resolver el problema relajado, el cual es típicamente realizado en la primera etapa de la optimización mixta entera. De acuerdo a los resultados, el tiempo requerido para resolver problemas con mayor número de pozos se incrementa de forma lineal. Sin embargo, el incremento del número de concentradores si incrementa significativamente el tiempo de solución. Afortunadamente, el número de concentradores es usualmente bajo en comparación con la gran cantidad de pozos que interconecta. Así, el tiempo de solución en la planeación de la producción usando el método propuesto en este trabajo siempre será menor que el requerido en una programación mixta entera.

\section{CONCLUSIONES}

Se ha desarrollado un modelo capaz de optimizar la planeación de la producción del gas y petróleo en diferentes periodos de tiempo. El tamaño del período debe ser lo suficiente para garantizar que las premisas tales como valores razonablemente constantes del flujo producido sean garantizadas. El modelo considera restricciones de producción del yacimiento debido a sus propiedades geológicas y de la tubería utilizada para el transporte del fluido del yacimiento al punto de entrega minimizando el costo de producción. El modelo demuestra que es posible resolver este tipo de problemas sin usar la estrategia de la programación mixta entera en la que se introducen variables binarias que incrementan el tiempo computacional para llegar a la solución (Robles-Agudo et al., 2007).

\section{AGRADECIMIENTOS}

Los autores agradecen el apoyo de la DGEST, CONCYTEG, CONACYT y PIFI.

\section{REFERENCIAS}

Barragán-Hernández, V., R. Vázquez-Román, L. Rosales-Marines y F. García-Sánchez; A Strategy for Simulation and Optimization of Gas and Oil Production, Computers and Chemical Engineering: 30(2), 215-227 (2005).

Beggs, H.D.; Production Optimization using NODALTM analysis, 2a edición, OGCI Inc., Tulsa, OK, (2003).

Brooke, A., D. Kendrick, A. Meeraus y R. Raman; GAMS- a user guide, Washington, DC, USA: GAMS Development Corporation (1998).

Economides, M.J., A.D. Hill y C. Ehlig-Economides; Petroleum production systems, Prentice Hall Petroleum Engineering Series, USA (1994).

Eeg, O.S. y T. Herring; Combining linear programming and reservoir simulation to optimize asset value, In: Paper SPE 37446(1997). 
Green, D.W. y G.P. Willhite; Enhanced oil recovery, SPE Textbook Series, Volume 6, USA (1998).

Handley-Schachler, S., C. McKie y N. Quintero; New Mathematical Techniques for the Optimization of Oil and Gas production Systems, In: Paper SPE 65161(2000).

Heinemann, R.F., S.L. Lyons y W. Tai; Next generation reservoir optimization, World Oil: 219(1), 4754 (1998).

Horne, R.N.; Modern well test analysis, 2a edición, Palo Alto, CA, USA: Petroway Inc, (1998).

Iyer, R.R., I.E. Grossmann, S. Vasantharajan y A.S. Cullick; Optimal planning and scheduling of offshore oil field infrastructure investment and operations, Industrial and Engineering Chemistry Research: 37(4), 1380-1397 (1998).

Kosmidis, V.D., J.D. Perkins y E.N. Pistikopoulos; Optimization of Well Oil Rate Allocations in Petroleum Fields, Industrial and Engineering Chemistry Research: 43(14), 3513 - 3527 (2004).

Kosmidis, V.D., J.D. Perkins y E.N. Pistikopoulos; A mixed integer optimization formulation for the well scheduling problem on petroleum fields, Computers \& Chemical Engineering: 29(7), 1523-1541 (2005).

Ortíz-Gómez, A., V. Rico-Ramírez y R. Vazquez-Roman; Mixed-integer multiperiod model for the planning of oilfield production European Symposium on Computer Aided Process Engineering-11, 907-912 R. Gani and S.B. Jorgensen (Edts), Elsevier Science(2001).

Robles-Agudo, O., R. Vázquez-Román, I.E. Grossmann y G. Iglesias-Silva; A multiperiod planning model for the oil and gas production system, Computers \& Chemical Engineering: Enviado para su publicación (2007).

Tapabrata, R. y R. Sarker; Optimum oil production planning using an evolutionary approach, Studies in Computational Intelligence: 49, 273-292 (2007).

Ulstein, N.L., B. Nygreen y J.R. Sagli; Tactical planning of offshore petroleum production, European Journal of Operational Research: 176(1), 550-564 (2007).

Van den Heever, S.A. y I.E. Grossmann; An iterative aggregation/disaggregation approach for the solution of a mixed-integer nonlinear oilfield infrastructure planning model, Industrial and Engineering Chemistry Research: 39(6), 1955-1971 (2000).

Van den Heever, S.A., I.E. Grossmann, S. Vasantharajan y K. Edwards; A Lagrangean Decomposition Heuristic for the Design and Planning of Offshore Hydrocarbon Field Infrastructures with Complex Economic Objectives, Industrial and Engineering Chemistry Research: 40(13), 2857-2875 (2001).

Vázquez-Román, R.; Predicción de la Presión y Temperatura en Tuberías de Extracción de Gas, Información Tecnológica: 8(4), 187-194 (1998).

Zhu, Y., H. Weng, Z. Chen y Q. Chen; Compositional modification of crude oil during oil recovery, Journal of Petroleum Science and Engineering: 38(1-2), 1-11 (2003). 